Supporting Information

\title{
A Zirconium Hydride Complex Supported by a Tetradentate Carbon-Centered Tripodal Tris(aryloxide) Ligand: Synthesis, Structure, and Reactivity
}

\author{
Yusuke Nakanishi, Yutaka Ishida and Hiroyuki Kawaguchi* \\ Department of Chemistry, Tokyo Institute of Technology, \\ Ookayama, Meguro-ku, Tokyo 152-8551, Japan \\ hkawa@chem.titech.ac.jp
}

Table of Contents

$\begin{array}{ll}\text { Additional NMR Spectroscopic Data } & \text { S2 }\end{array}$

$\begin{array}{ll}\text { Crystallographic Data } & \text { S7 }\end{array}$ 


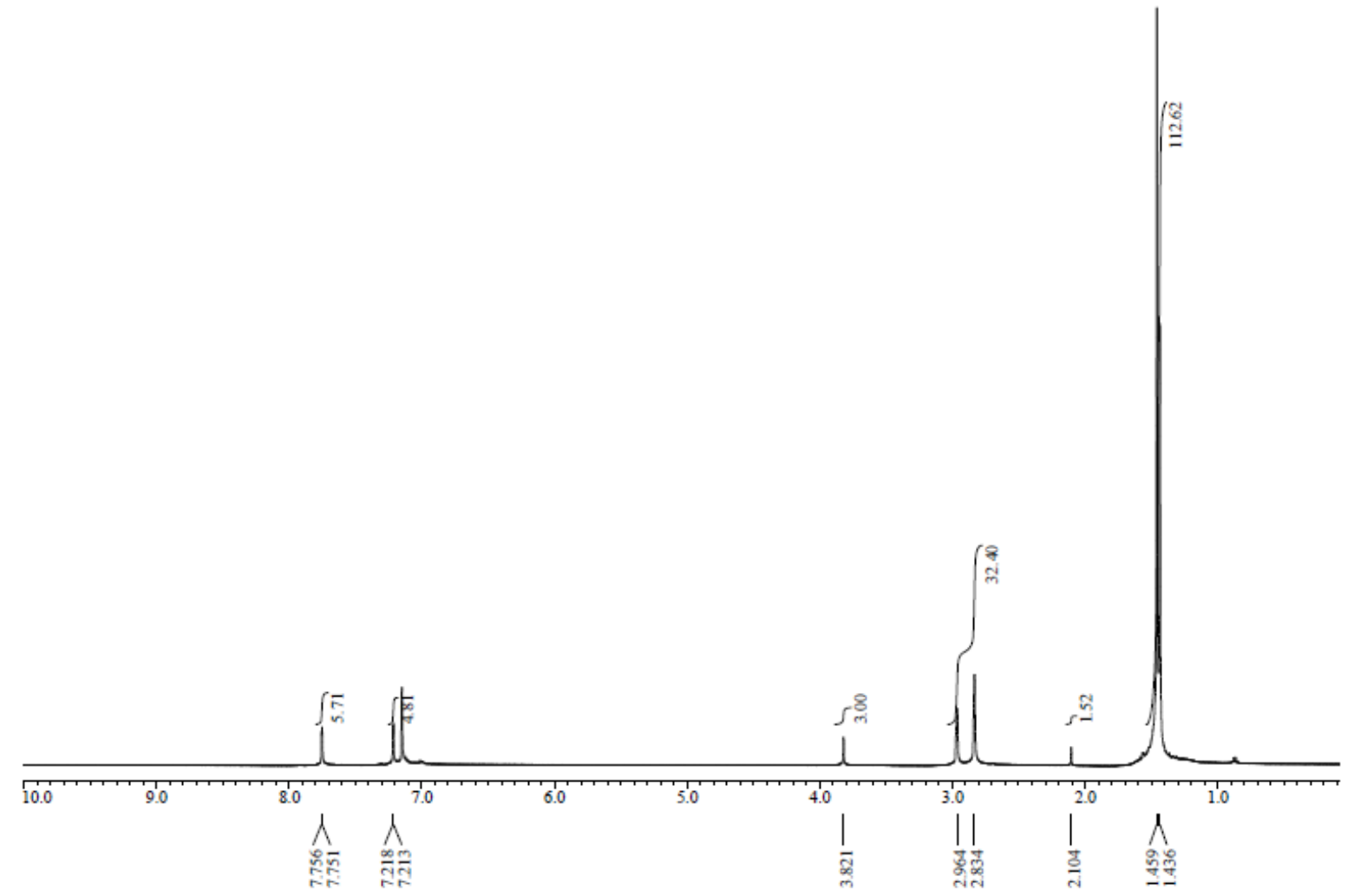

Figure S1. ${ }^{1} \mathrm{H}-\mathrm{NMR}$ spectrum of $\mathbf{2} \cdot\left(\mathbf{C}_{\mathbf{7}} \mathbf{H}_{\mathbf{8}}\right)_{0.5}$ in $\mathrm{C}_{6} \mathrm{D}_{6}$. 


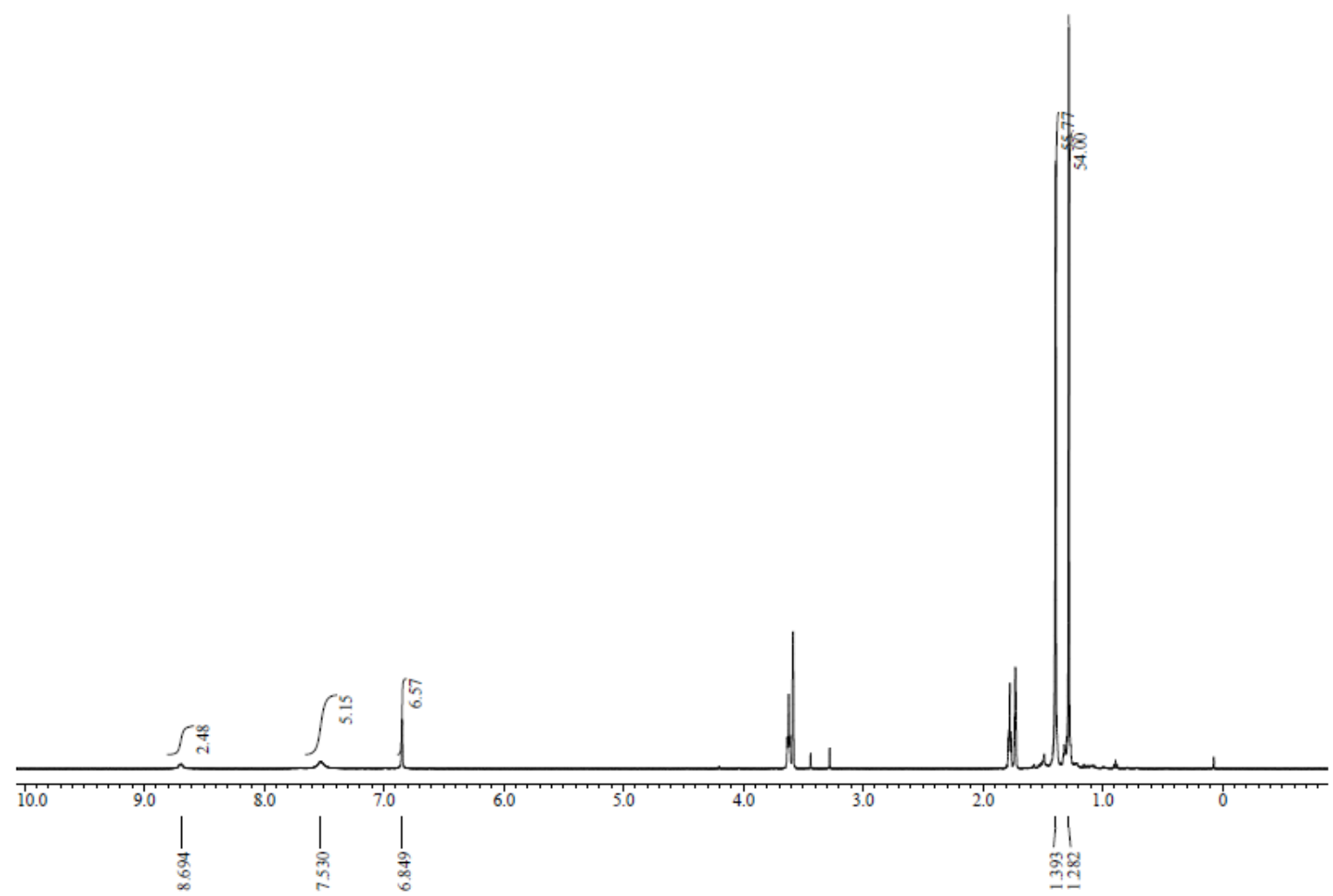

Figure S2. ${ }^{1} \mathrm{H}-\mathrm{NMR}$ spectrum of $\mathbf{3}$ in THF- $d_{8}$ at $333 \mathrm{~K}$. 


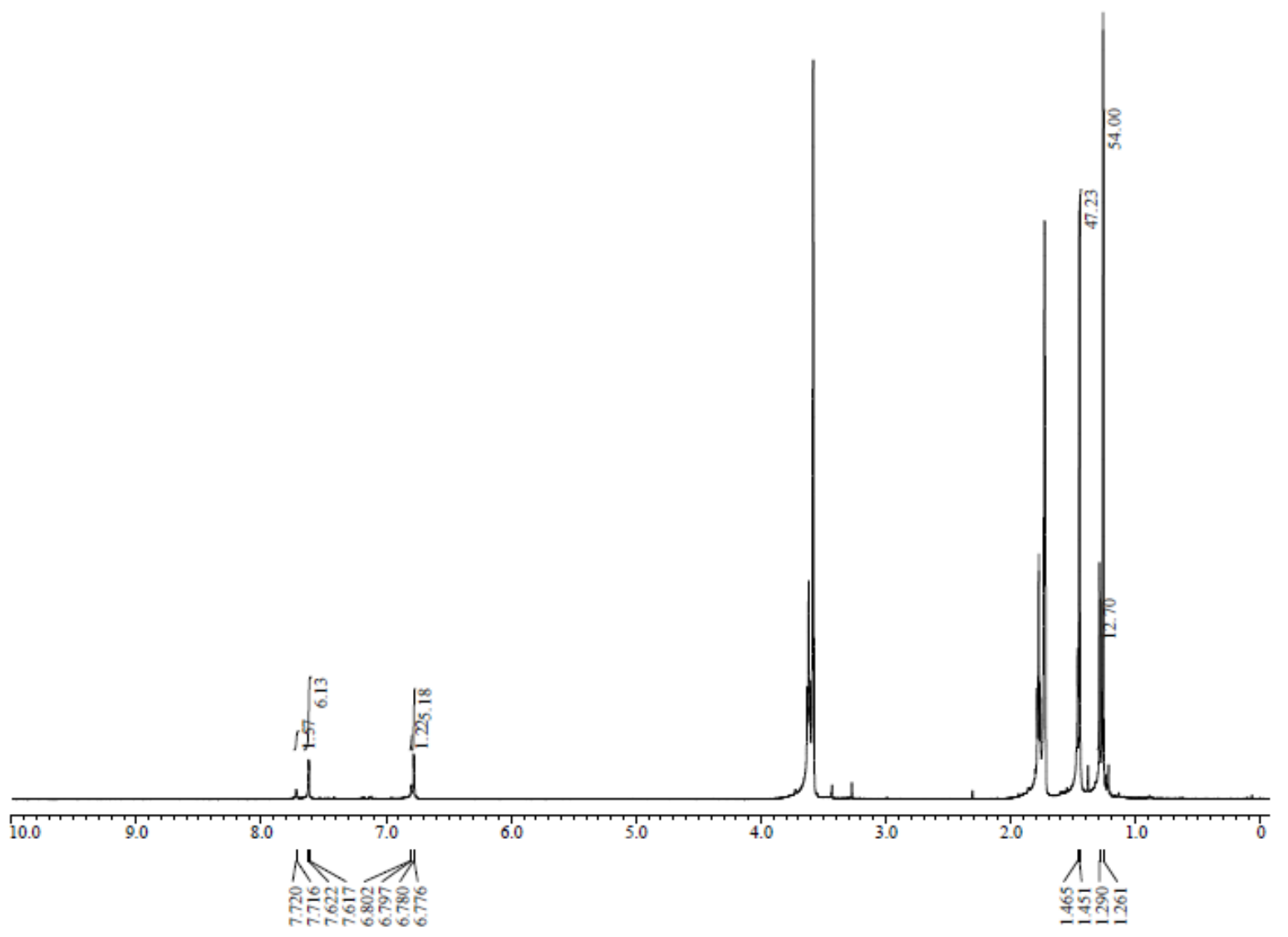

Figure S3. ${ }^{1} \mathrm{H}-\mathrm{NMR}$ spectrum of $\mathbf{4}$ in THF- $d_{8}$. 


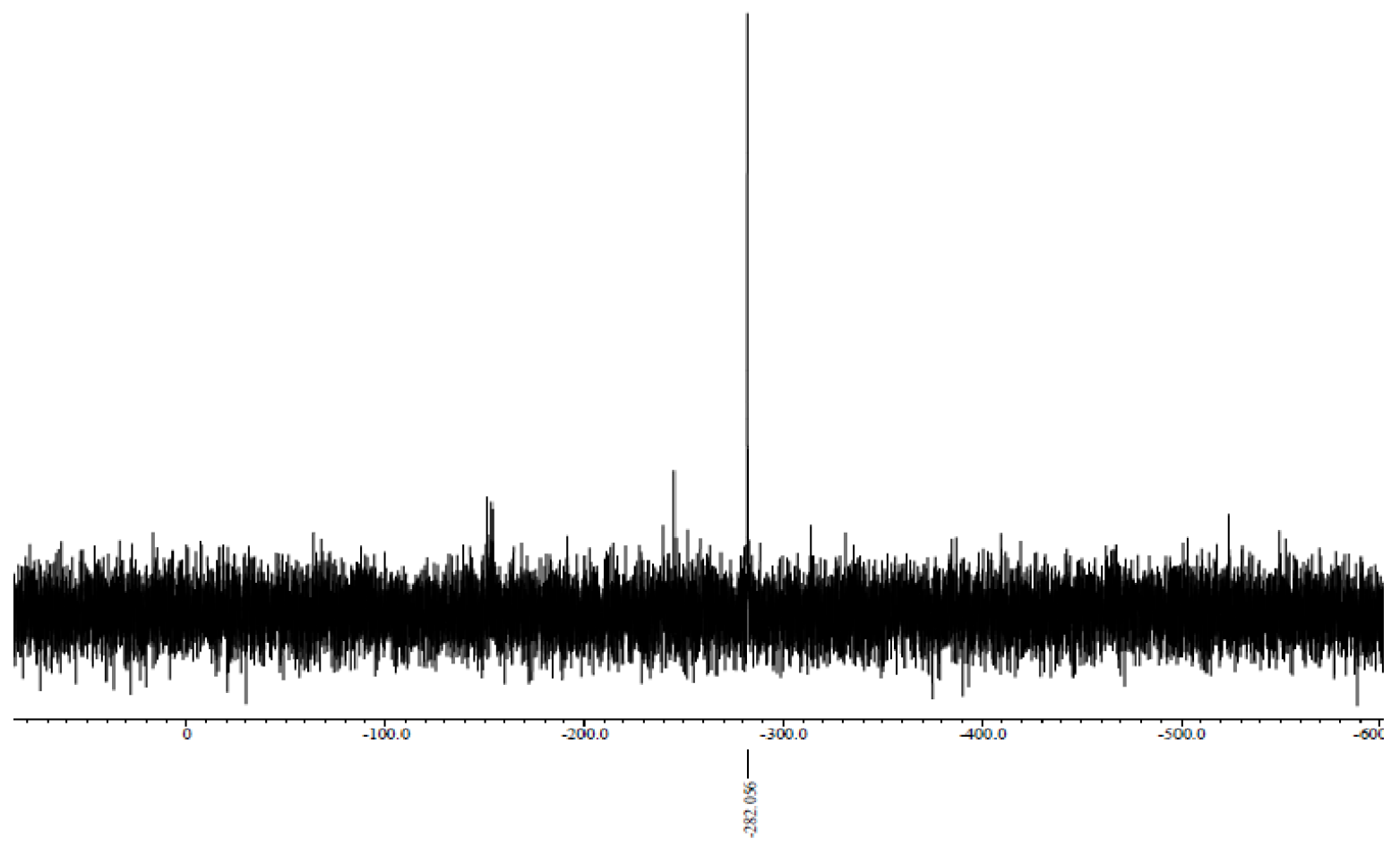

Figure $\mathbf{S 4} .{ }^{31} \mathrm{P}\left\{{ }^{1} \mathrm{H}\right\}$ NMR spectrum of $\mathbf{4}$ in THF- $d_{8}$. 


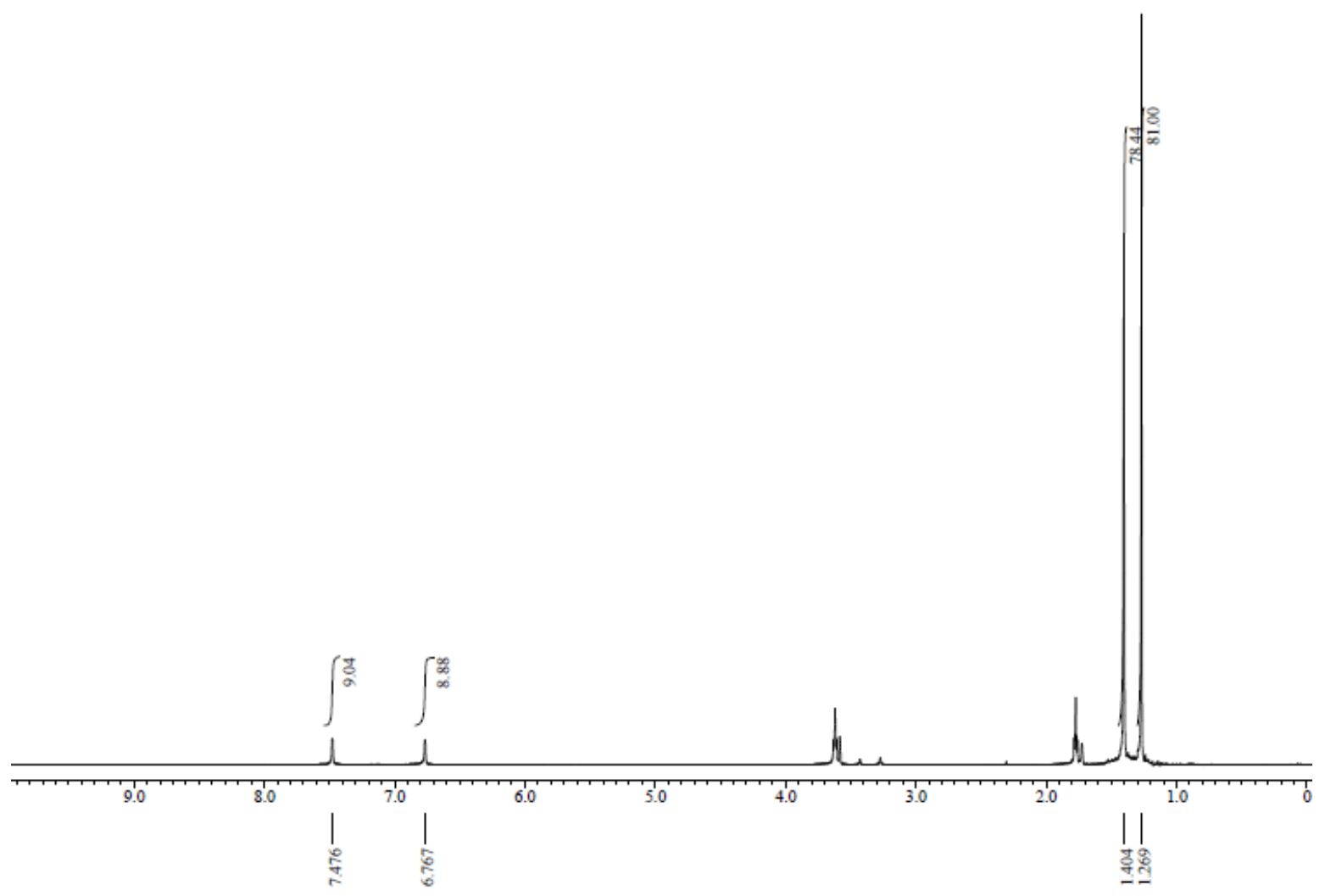

Figure S5. ${ }^{1} \mathrm{H}$ NMR spectrum of 5 in THF- $d_{8}$. 
Table S1. Crystal Data for 2

\begin{tabular}{|c|c|}
\hline formula & $\mathrm{C}_{98} \mathrm{H}_{153} \mathrm{O}_{12} \mathrm{Zr}_{2} \mathrm{Na}_{3} \cdot 2\left(\mathrm{C}_{7} \mathrm{H}_{8}\right)$ \\
\hline $\operatorname{mol~wt}\left(\mathrm{g} \mathrm{mol}^{-1}\right)$ & 1958.87 \\
\hline$T(\mathrm{~K})$ & $123(2)$ \\
\hline crystal system & monoclinic \\
\hline space group & $P 2{ }_{1} / c(\# 14)$ \\
\hline crystal color & Yellow \\
\hline crystal size (mm) & $0.10 \times 0.08 \times 0.06$ \\
\hline Solvent & DME/toluene \\
\hline$a(\AA)$ & $20.449(2)$ \\
\hline$b(\AA)$ & $32.406(3)$ \\
\hline$c(\AA)$ & $17.828(2)$ \\
\hline$\alpha(\operatorname{deg})$ & 90 \\
\hline$\beta(\operatorname{deg})$ & $112.5180(10)$ \\
\hline$\gamma(\operatorname{deg})$ & 90 \\
\hline$V\left(\AA^{3}\right)$ & $10913(2)$ \\
\hline $\mathrm{Z}$ & 4 \\
\hline$\rho_{\text {calc }}\left(\mathrm{g} \mathrm{cm}^{-3}\right)$ & 1.192 \\
\hline$\mu(\operatorname{Mo~K\alpha })\left(\mathrm{cm}^{-1}\right)$ & 0.259 \\
\hline Abs. Correction type & Empirical \\
\hline Abs. Transmission (min/max) & $0.8307 / 1.0000$ \\
\hline $2 \theta_{\max }(\mathrm{deg})$ & 54.9 \\
\hline no. of reflections collected & 76808 \\
\hline no. of unique reflections $\left(\mathrm{R}_{\text {int }}\right)$ & $24913(0.0566)$ \\
\hline$R_{1}[\mathrm{I}>2 \sigma(I)]^{\mathrm{a}}$ & 0.0783 \\
\hline $\mathrm{w} R_{2}$ (all data) ${ }^{\mathrm{b}}$ & 0.2177 \\
\hline Goodness of fit on $F^{2}$ & 1.077 \\
\hline Largest diff. peak and hole/e $\AA^{-3}$ & 1.731 and $-0.842 \mathrm{e} \cdot \AA^{-3}$ \\
\hline
\end{tabular}


Table S2. Crystal Data for 3

\begin{tabular}{|c|c|}
\hline formula & $\mathrm{C}_{113} \mathrm{H}_{171} \mathrm{O}_{18} \mathrm{Zr}_{2} \mathrm{Na}_{3} \cdot\left(\mathrm{C}_{4} \mathrm{H}_{8} \mathrm{O}\right) \cdot\left(\mathrm{C}_{5} \mathrm{H}_{12}\right)$ \\
\hline $\operatorname{mol~wt}\left(\mathrm{g} \mathrm{mol}^{-1}\right)$ & 2213.15 \\
\hline$T(\mathrm{~K})$ & $123(2)$ \\
\hline crystal system & monoclinic \\
\hline space group & $P 2_{1}(\# 4)$ \\
\hline crystal color & Colorless \\
\hline crystal size $(\mathrm{mm})$ & $0.331 \times 0.07 \times 0.06$ \\
\hline Solvent & pentane/THF \\
\hline$a(\AA)$ & $15.356(3)$ \\
\hline$b(\AA)$ & $19.003(4)$ \\
\hline$c(\AA)$ & $21.354(4)$ \\
\hline$\alpha(\operatorname{deg})$ & 90 \\
\hline$\beta(\operatorname{deg})$ & $90.029(4)$ \\
\hline$\gamma(\mathrm{deg})$ & 90 \\
\hline$V\left(\AA^{3}\right)$ & $6231(2)$ \\
\hline Z & 2 \\
\hline$\rho_{\text {calc }}\left(\mathrm{g} \mathrm{cm}^{-3}\right)$ & 1.180 \\
\hline$\mu(\mathrm{Mo} \mathrm{K} \alpha)\left(\mathrm{cm}^{-1}\right)$ & 0.238 \\
\hline Abs. Correction type & Empirical \\
\hline Abs. Transmission $(\min / \max )$ & $0.8663 / 1.0000$ \\
\hline $2 \theta_{\max }(\operatorname{deg})$ & 54.9 \\
\hline no. of reflections collected & 56666 \\
\hline no. of unique reflections $\left(\mathrm{R}_{\text {int }}\right)$ & $28321(0.0459)$ \\
\hline$R_{1}[\mathrm{I}>2 \sigma(I)]^{\mathrm{a}}$ & 0.0701 \\
\hline $\mathrm{w} R_{2}$ (all data) ${ }^{\mathrm{b}}$ & 0.1869 \\
\hline Goodness of fit on $F^{2}$ & 1.075 \\
\hline Largest diff. peak and hole/e $\AA^{-3}$ & 0.958 and $-0.971 \mathrm{e} \cdot \AA^{-3}$ \\
\hline
\end{tabular}


Table S3. Crystal Data for 4

\begin{tabular}{|c|c|}
\hline formula & $2\left(\mathrm{C}_{20} \mathrm{H}_{40} \mathrm{NaO}_{5}\right) \cdot 2\left(\mathrm{C}_{102} \mathrm{H}_{152} \mathrm{Na}_{2} \mathrm{O}_{10} \mathrm{P}_{3} \mathrm{Zr}_{2}\right) \cdot 5\left(\mathrm{C}_{4} \mathrm{H}_{8} \mathrm{O}\right)$ \\
\hline $\operatorname{mol~wt}\left(\mathrm{g} \mathrm{mol}^{-1}\right)$ & 4846.65 \\
\hline$T(\mathrm{~K})$ & $123(2)$ \\
\hline crystal system & monoclinic \\
\hline space group & $P c(\# 7)$ \\
\hline crystal color & Yellow \\
\hline crystal size $(\mathrm{mm})$ & $0.20 \times 0.17 \times 0.12$ \\
\hline Solvent & THF \\
\hline$a(\AA)$ & $23.381(5)$ \\
\hline$b(\AA)$ & $14.740(3)$ \\
\hline$c(\AA)$ & $40.457(9)$ \\
\hline$\alpha(\operatorname{deg})$ & 90 \\
\hline$\beta(\operatorname{deg})$ & $94.695(2)$ \\
\hline$\gamma(\operatorname{deg})$ & 90 \\
\hline$V\left(\AA^{3}\right)$ & $13896(5)$ \\
\hline $\mathrm{Z}$ & 2 \\
\hline$\rho_{\text {calc }}\left(\mathrm{g} \mathrm{cm}^{-3}\right)$ & 1.158 \\
\hline$\mu(\mathrm{Mo} \mathrm{K} \alpha)\left(\mathrm{cm}^{-1}\right)$ & 0.251 \\
\hline Abs. Correction type & Empirical \\
\hline Abs. Transmission $(\min / \max )$ & $0.9105 / 1.0000$ \\
\hline $2 \theta_{\max }(\operatorname{deg})$ & 54.9 \\
\hline no. of reflections collected & 151258 \\
\hline no. of unique reflections $\left(\mathrm{R}_{\mathrm{int}}\right)$ & $62025(0.0735)$ \\
\hline$R_{1}[\mathrm{I}>2 \sigma(I)]^{\mathrm{a}}$ & 0.0918 \\
\hline $\mathrm{w} R_{2}$ (all data) ${ }^{\mathrm{b}}$ & 0.2321 \\
\hline Goodness of fit on $F^{2}$ & 1.037 \\
\hline Largest diff. peak and hole/e $\AA^{-3}$ & 0.963 and $-0.612 \mathrm{e} \cdot \AA^{-3}$ \\
\hline
\end{tabular}


Table S4. Crystal Data for 5

\begin{tabular}{|c|c|}
\hline formula & $\mathrm{C}_{165} \mathrm{H}_{252} \mathrm{~N}_{9} \mathrm{Na}_{3} \mathrm{O}_{18} \mathrm{Zr}_{3} \bullet 4\left(\mathrm{C}_{5} \mathrm{H}_{12}\right)$ \\
\hline $\operatorname{mol~wt}\left(\mathrm{g} \mathrm{mol}^{-1}\right)$ & 3425.25 \\
\hline$T(\mathrm{~K})$ & $123(2)$ \\
\hline crystal system & cubic \\
\hline space group & $P a-3(\# 205)$ \\
\hline crystal color & Colorless \\
\hline crystal size (mm) & $0.21 \times 0.15 \times 0.14$ \\
\hline Solvent & pentane/THF \\
\hline$a(\AA)$ & $34.4443(9)$ \\
\hline$b(\AA)$ & $34.4443(9)$ \\
\hline$c(\AA)$ & $34.4443(9)$ \\
\hline$\alpha(\operatorname{deg})$ & 90 \\
\hline$\beta(\operatorname{deg})$ & 90 \\
\hline$\gamma(\operatorname{deg})$ & 90 \\
\hline$V\left(\AA^{3}\right)$ & $40865(3)$ \\
\hline $\mathrm{Z}$ & 8 \\
\hline$\rho_{\text {calc }}\left(\mathrm{g} \mathrm{cm}^{-3}\right)$ & 1.067 \\
\hline$\mu(\operatorname{Mo~K} \alpha)\left(\mathrm{cm}^{-1}\right)$ & 0.212 \\
\hline Abs. Correction type & Empirical \\
\hline Abs. Transmission $(\min / \max )$ & $0.8540 / 1.0000$ \\
\hline $2 \theta_{\max }(\mathrm{deg})$ & 54.9 \\
\hline no. of reflections collected & 334006 \\
\hline no. of unique reflections $\left(\mathrm{R}_{\mathrm{int}}\right)$ & $15552(0.1648)$ \\
\hline$R_{1}[\mathrm{I}>2 \sigma(I)]^{\mathrm{a}}$ & 0.994 \\
\hline $\mathrm{w} R_{2}$ (all data) ${ }^{\mathrm{b}}$ & 0.3210 \\
\hline Goodness of fit on $F^{2}$ & 1.105 \\
\hline Largest diff. peak and hole/e $\AA^{-3}$ & 1.386 and $-0.554 \mathrm{e} \cdot \AA^{-3}$ \\
\hline
\end{tabular}

